\title{
Numerical Simulation of Flow over an Open Cavity with Self-Sustained Oscillation Mode Switching
}

\author{
Takashi Yoshida1 ${ }^{*}$, Takashi Watanabe ${ }^{2}$ \\ ${ }^{1}$ Department of Mechanical Systems Engineering, Shinshu University, Nagano, Japan \\ ${ }^{2}$ Graduate School of Information and Science, Nagoya University, Nagoya, Japan \\ Email: *yoshi-t@shinshu-u.ac.jp
}

How to cite this paper: Yoshida, T. and Watanabe, T. (2016) Numerical Simulation of Flow over an Open Cavity with SelfSustained Oscillation Mode Switching. Open Journal of Fluid Dynamics, 6, 361-370. http://dx.doi.org/10.4236/ojfd.2016.64027

Received: November 8, 2016

Accepted: December 17, 2016

Published: December 20, 2016

Copyright $\odot 2016$ by authors and Scientific Research Publishing Inc. This work is licensed under the Creative Commons Attribution International License (CC BY 4.0).

http://creativecommons.org/licenses/by/4.0/ (c) (i) Open Access

\begin{abstract}
Numerical simulations are used to investigate the self-sustained oscillating flows past an open cavity. The two-dimensional incompressible Navier-Stokes equations are solved directly by using the finite difference method for cavities with an upstream laminar boundary layer. A series of simulations are performed for a variety of cavity length-to-depth ratio. The results show the switching among some flow modes including non-oscillation mode, shear layer mode and wake mode. The variation of the Strouhal number is in favorable agreement with available experimental data. The results of flow fields in the cavity reveal the relationship between the cavity shear layer oscillation modes and recirculating vortices in the cavity.
\end{abstract}

\section{Keywords}

Open Cavity Flow, Self-Sustained Oscillations, Incompressible Flow, Numerical Simulation

\section{Introduction}

Flows over open cavities occur in a wide variety of aerospace and engineering applications, for example, the landing systems of aircrafts, sunroofs and windows of automobiles, and spaces between bullet train cars. A schematic of the cavity model and the cavity flow is illustrated in Figure 1 where the length and the depth of the cavity are $L$ and $D$, respectively. Cavity flow is of interest, because the presence of cavity causes selfsustained oscillations of the separated shear layer by a complex feedback mechanism, despite its geometrical simplicity. Rockwell and Naudascher [1] classified the flowinduced cavity oscillations and the feedback mechanism into fluid-dynamic and fluidresonant. Incompressible flows such as low-Mach number air flows, low-speed water flows over an open cavity are classified as fluid-dynamic oscillations. For this condition, 


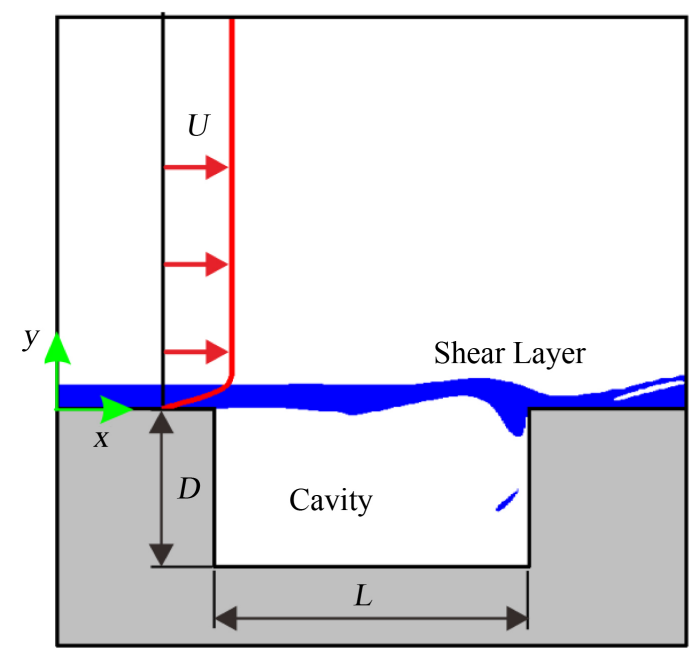

Figure 1. Schematic of the computational domain and the coordinates.

the acoustic wavelength is much longer than the length of the cavity, so that pressure fluctuations propagate instantaneously to the upstream leading edge of the cavity. The feedback mechanism can be regarded as purely hydrodynamic. The self-sustaining cavity oscillations in compressible flows at high Mach numbers are classified as fluidresonant oscillations. The acoustic wavelength is of the same order of magnitude as the cavity length. The acoustic pressure disturbances radiate and propagate toward the upstream edge with acoustic speed and there is an acoustic delay. The flow-acoustic resonance arises from this feedback loop. This feedback mechanism can regard as acoustic.

It is well known that the primary frequency of shear layer oscillations varies with cavity length. Many experimental studies have been carried out to reveal the characteristics of the frequency variation (Sarohia [2], Knisely and Rockwell [3], Gharib [4], Gharib and Roshko [5]). The general feature of the variation of dominant frequency represented by the Strouhal number $S t$ with the length-to-depth ratio $L / D$ is shown in Figure 2. A minimum length-to-depth ratio is required for the onset of self-sustained oscillations. When the ratio exceeds some value, the "shear layer mode" appears, there the flow oscillation is found. In this mode, first, the Strouhal number decreases as $L / D$. However, the Strouhal number jumps to higher value at the critical length-to-depth ratio. This critical ratio divides the shear layer mode into two modes, and these modes at the lower and the higher length-to-depth ratio are called mode II and mode III, respectively. With a further increase of the cavity length, another mode has been observed. Gharib and Roshko [5] used the term "wake mode" to describe this mode, since the flow is characterized by large scale vortex shedding from the cavity to the freestream region, which looks like the wake behind a bluff body.

Unlike the experimental studies, the numerical studies of mode switching among the mode II and mode III and the wake mode so far have been limited to few papers. Rowley et al. [6] investigated the shear layer mode and wake mode in the two-dimensional 


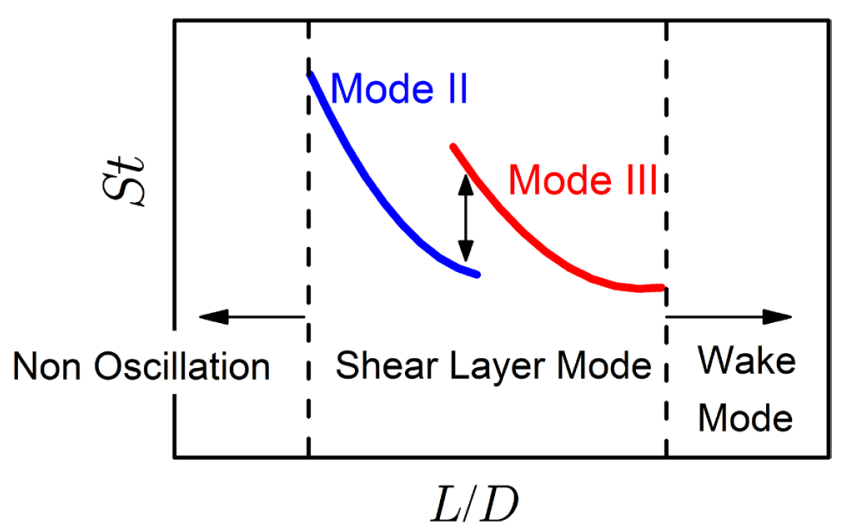

Figure 2. Schematic of Strouhal number variation and the definition of the cavity oscilation modes.

subsonic flow for $L / D=1,2,3,4,5$ using the two-dimensional direct numerical simulation. Rubio et al. [7] investigated the two different modes in subsonic flow for $L / D=2$, 3, 4 using the two-dimensional large eddy simulation. However, no detailed numerical analysis has been reported the mode switching for the incompressible cavity flow.

In this paper, we investigate numerically the mode switching in the two-dimensional incompressible flow over a rectangular open cavity. We perform the two-dimensional incompressible Navier-Stokes direct numerical simulations using the finite difference method. We also reveal the relationship between the cavity shear layer oscillations and recirculating vortices in the cavity.

\section{Numerical Method}

\subsection{Numerical Implementation}

Schematic of the computation domain is shown in Figure 1. The governing equations are the two-dimensional, unsteady, incompressible Navier-Stokes equations and the equation of continuity in Cartesian coordinates $(x, y)$. The origin is at the most upstream point on the wall. The velocity components are $(u, v)$ in the directions $(x, y)$. All variables are nondimensionalized using the cavity depth $D$ and the free-stream velocity $U$. These equations are integrated in time using the P2 pressure correction method by Armfield and Street [8]. The momentum equations are discretized using the second order Adams-Bashforth method for the convective terms and the Crank-Nicolson method for the diffusive terms. These equations are solved by the fractional step method to enforce the solenoidal condition. The pressure correction term is used with the Kim and Moin type boundary condition [9] in order to reduce the projection error and recover the second-order accuracy in time. The resulting linear algebraic systems are solved by the biconjugate gradient stabilized method. Nonuniform staggered grid systems, which cluster node points in the boundary layer, the shear layer, the cavity bottom, and the cavity edges, are used for the spatial descretization. The second order fully conservative finite difference scheme by Morinishi et al. [10] is used for the convective terms and the second order central difference scheme was used for the other terms. 
In Figure 1, the computational domain extends to $5 D$ upstream of the cavity leading edge, $7 D$ downstream of the trailing edge and $9 D$ in the normal direction above the cavity. This dimension of the computational domain is similar to that used in the two-dimensional simulations by Rowley et al. [6]. The laminar Blasius boundary layer is specified in the inflow boundary. A free-slip condition is applied to the top boundary and the no-slip boundary condition is applied to the wall. At the outflow boundary, we use the Sommerfeld radiation condition, which is also called the convective outflow condition. The convective velocity in this condition is set equal to the free-stream velocity $U$. This boundary condition allows vortices to smoothly pass across the computational domain. The Reynolds number based on the free-stream velocity $U$ and the cavity depth is 6000. The boundary layer momentum thickness $\theta$ at the inflow boundary is set to 0.00268 . This value of the momentum thickness results in the momentum thickness at the upstream edge of the cavity to be 0.0322 for $L / D=2.0$. The Reynolds number estimated from the momentum thickness of 0.0322 and the free-stream velocity is 193, which is similar to 190 used in the experiment by Knisely and Rockwell [3].

\subsection{Grid Refinement Study}

The computational results are validated by performing systematic grid refinement studies to ensure that the results are independence of grid resolution. The case of $L / D=$ 2.0 is calculated with five grids shown in Table 1. Figure 3 shows time variations of the

Table 1. Grid points of deferent grids.

\begin{tabular}{lcc}
\hline & Grid points in the cavity & Total grid points \\
\hline Grid 1 & $56 \times 19$ & $111 \times 50$ \\
Grid 2 & $113 \times 38$ & $223 \times 100$ \\
Grid3 & $225 \times 75$ & $445 \times 200$ \\
Grid 4 & $338 \times 113$ & $668 \times 300$ \\
Grid 5 & $450 \times 150$ & $890 \times 400$ \\
\hline
\end{tabular}

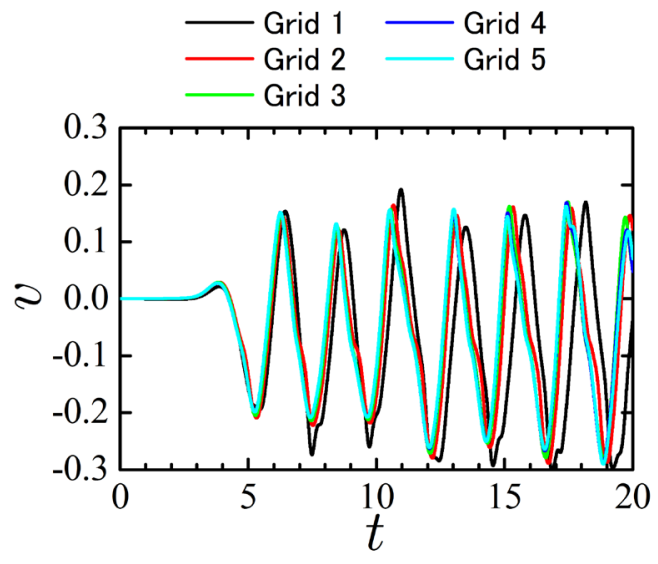

Figure 3. Effect of the grid resolution on the time variations of the $y$ velocity component $v$ at $x=6.9$, $y=0.0$ and $L / D=2.0$. 
$y$ velocity component $v$ near the downstream edge of cavity $(x=6.9, y=0.0)$. The results of grids finer than Grid 3 are almost identical.

Figure 4 shows the spectra of $v$ during periodic oscillations. The most dominant peak and other harmonic peaks predicted by the grids finer than Grid 3 are in very good agreement. From these tests, the results obtained on the grids finer than Grid 3 are independent of grid size. We employ Grid 3 for sufficient accuracy.

\section{Results and Discussion}

\subsection{Mode Switching}

A series of two-dimensional simulations for varying the length-to-depth ratio $L / D$ from 1.0 to 4.0 at 0.1 interval have been conducted. The shear layer mode is characterized by periodic oscillations of separated shear layer. The Strouhal number is defined by $S t=$ $f \theta / U$, where $f$ is the frequency of the most dominant oscillation. The Strouhal number corresponds to the frequency of the most dominant peak. Figure 5 shows the Strouhal number variation with cavity length, as well as experimental data obtained by Knisely and Rockwell [3]. The cavity length is normalized with respect to the reference momentum thickness at the upstream edge of cavity $\theta$ and the Strouhal number is based on the same $\theta$ for comparison to experimental data. No shear layer oscillation occur below $L / D=1.6(L / \theta=50.0)$. The minimum length for initiation of self-sustained oscillations is $L / D=1.7(L / \theta=52.7)$. The Strouhal number decreases as $L / D$ increases. This variation is interrupted by a sudden jump to a higher value between $L / D=3.1(L / \theta$ $=96.2)$ and $L / D=3.2(L / \theta=99.3)$. The oscillation regime at $L / D \leq 3.1$ is called as mode II, while that at $3.2 \leq L / D$ is named mode III. The maximum length-to-depth ratio in mode III is $L / D=3.6(L / \theta=111.3)$. Up to this length-to-depth ratio, the present results obtained by the present two-dimensional simulation closely agree with the experimental data. When the length-to-depth ratio is $L / D=3.7$, the oscillation mode changes to

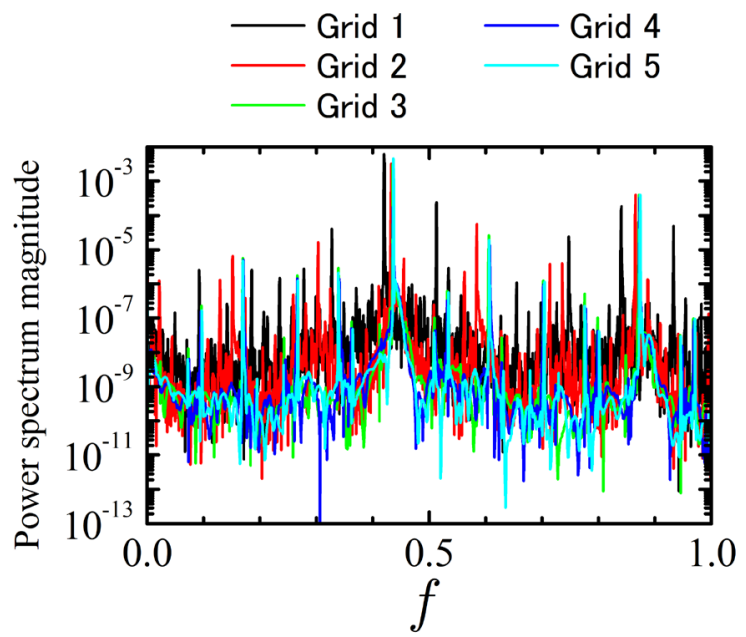

Figure 4. Effect of the grid resolution on the power spectra of the $y$ velocity component $v$ at $x=6.9, y=0.0$ and $L / D=2.0$. 


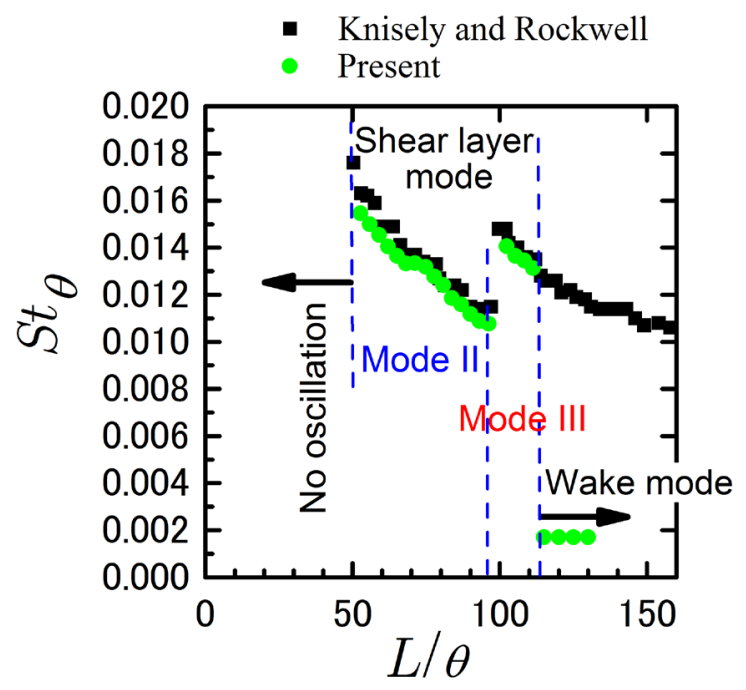

Figure 5. The Strouhal number variation with cavity length compared to experimental data [3].

the wake mode. The numerically obtained Strouhal number at $3.7 \leq L / D \leq 4.0$ is smaller than that in the mode III. As will be discussed in sec. 3.2 about the difference between the mode III and the wake mode predicted in the present study, the shear layer in the mode III oscillats on the multiple recirculating vortices in the cavity, while the flow in the wake mode has a vortex that expands to nearly entire cavity and sheds from the cavity in a long time period. Therefore, the Strouhal number in the wake mode becomes very low. It might be suggested that Knisely and Rockwell [3] has shown only the shear layer mode and they did not well capture the wake mode.

\subsection{Features of Flow Field}

Figure 6 contrasts the instantaneous vorticity fields for $L / D=2.0$ (mode II), $L / D=3.5$ (mode III) and $L / D=4.0$ (wake mode). The blue contour shows the separated shear layer. For $L / D=2.0$ in mode II, the vortex sheet of the separated shear layer rolls up and a large vortical structure is observed in the upstream region of the cavity trailing edge, as shown in Figure 6(a). There are two waves in the cavity shear layer. Figure 6(b) shows an instantaneous vorticity for $L / D=3.5$ in mode III. Three waves are found in the cavity. The shapes of the shear layers in mode II and mode III are consistent with the dye visualization of Figure 6 of Gharib and Roshko [5] and the dye visualization of Figure 12 and Figure 13 of Knisely and Rockwell [3]. In the wake mode, the separated shear layer forms a large scale vortex in the cavity. This large scale vortex makes a secondary counter-rotating vortex and then sheds and ejects from the cavity. Figure 6(c) shows the ejection found in the wake mode flow for $L / D=4.0$. Though the shear layer is disturbed by the ejection and the flow pattern is different from those in mode II and mode III, the shape of the shear layer is consistent with the dye visualization result of Figure 7 of Gharib and Roshko [5]. As mentioned in Knisely and Rockwell [3], the experimental flow visualization of the spanwise characteristics of the separated shear layer 


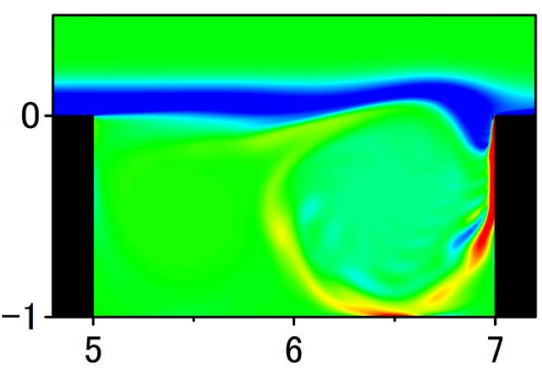

(a)

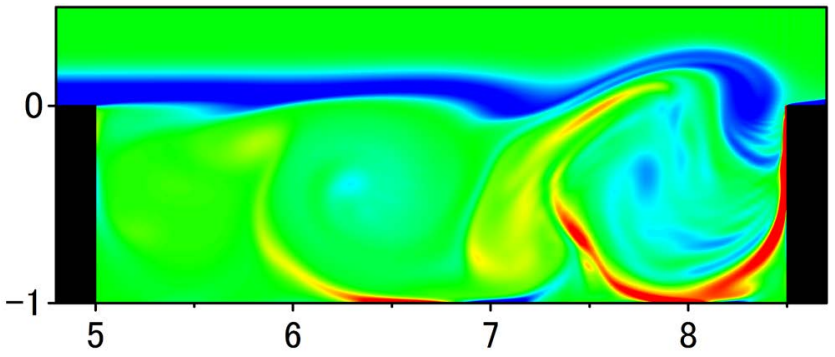

(b)

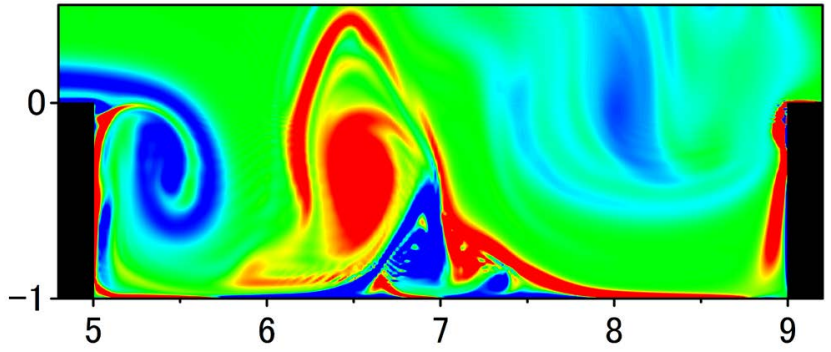

(c)

Figure 6. Instantaneous vorticity fields. (a) $L / D=2.0$. (b) $L / D=$ 3.5. (c) $L / D=4.0$.

showed a strong two-dimensional coherency for a wide range of the length-to-depth ratio. The experiment of Gharib and Roshko [5] were performed for an axisymmetric cavity mounted on a circular cylinder in an axial flow, and the flow visualization of the separated shear layer also showed two-dimensional characters. These facts strongly suggest that the mode switching concerned in this paper occurs in the two-dimensional space and the two-dimensional simulation is enough to investigate the flow.

Time-averaged streamlines for different values of the length-to-depth ratio are shown in Figure 7 to find the relationship between the mode switching and the recirculating vortices in the cavity. The blue lines indicate a clockwise vortex and the red lines indicate a counterclockwise vortex in the cavity. The flow field for non-oscillations cases, $L / D=1.0$ and $L / D=1.6$, are shown in Figure $7(\mathrm{a})$ and Figure $7(\mathrm{~b})$. The streamlines show a clockwise recirculating vortex which occupies the inside of the cavity. For $L / D=1.7$ in Figure 7 (c), which is the minimum cavity length for the initiation of the self-sustained oscillations in the study, a second counterclockwise vortex appears near the upstream edge of cavity and self-sustained oscillations starts. In Figure 7(d) for $L / D=$ 


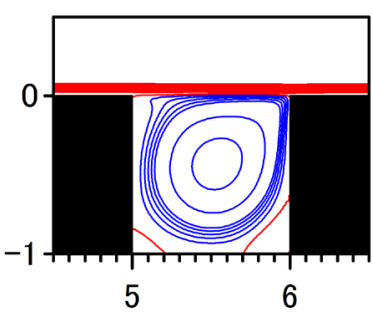

(a)

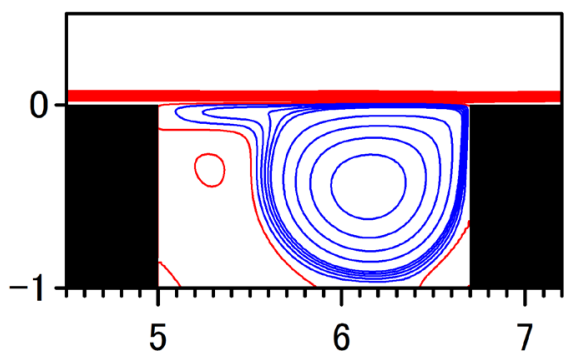

(c)

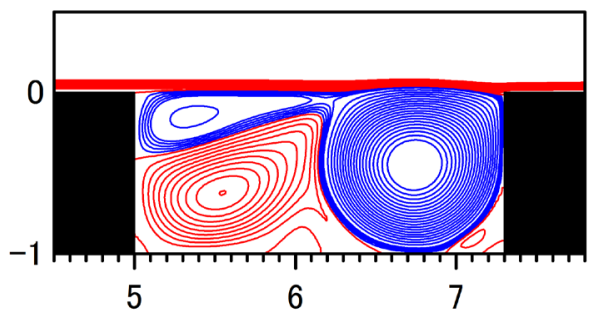

(e)

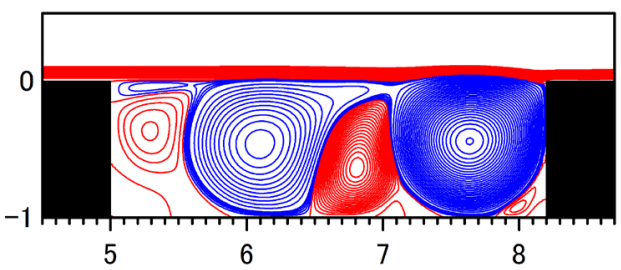

(g)

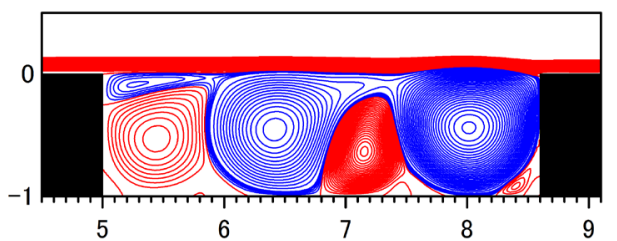

(i)

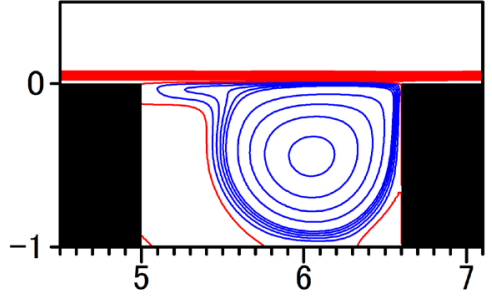

(b)

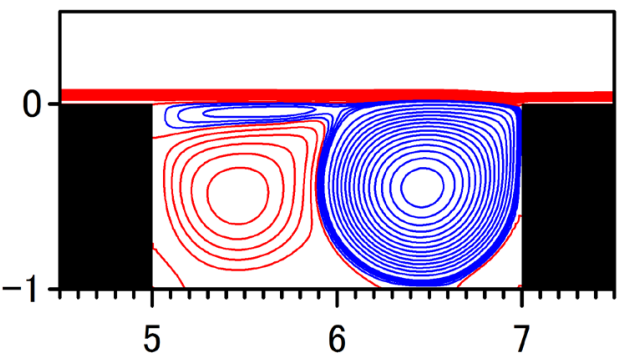

(d)

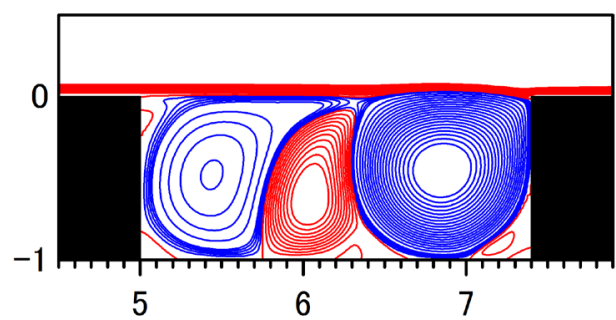

(f)

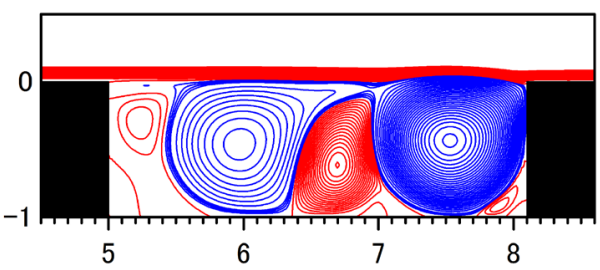

(h)

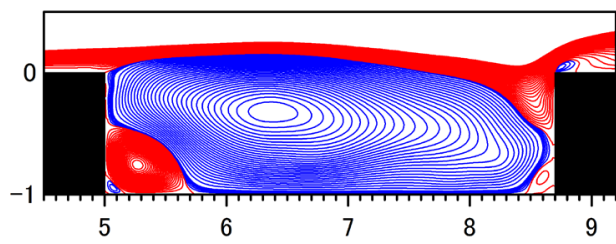

(j)

Figure 7. Time averaged streamlines for different values of the cavity length. (a) $L / D=1.0$. (b) $L / D=1.6$. (c) $L / D=1.7$ (mode II). (d) $L / D=2.0$. (e) $L / D=2.3$. (f) $L / D=2.4$. (g) $L / D=3.1$ (mode II). (h) $L / D=3.2$ (mode III). (i) $L / D=3.6$ (mode III). (j) $L / D=3.7$ (wake mode).

2.0, the counterclockwise vortex on the upstream side of the cavity becomes large and has similar scale as that of the clockwise vortex on downstream side of the cavity. The third clockwise thin vortex appears near the upstream edge of cavity. The third vortex becomes larger as the ratio is longer for $L / D=2.3$ in Figure $7(\mathrm{e})$. For $L / D=2.4$ in 
Figure 7(f), the third clockwise vortex is formed on the upstream side of the cavity. The Strouhal number decreases continuously, therefore the oscillations are in mode II. There are four vortices in the cavity for $L / D=3.1$, which is maximum length in mode II, as shown in Figure $7(\mathrm{~g})$. In Figure $7(\mathrm{~h})$ for $L / D=3.2$, a new clockwise thin vortex appears near the upstream edge of the cavity and the mode of oscillations switches from mode II to mode III. For $L / D=3.6$ in Figure 7(i), there are five vortices which are alternately rotating. For $L / D=3.7$ in Figure $7(j)$, the oscillation mode is the wake mode, then the large clockwise vortex appears in the cavity. The results indicate that the mode switching from non-oscillation occurs when the number of recirculating vortices changes from one to two. The mode switching from the mode II to the mode III occurs when the number of recirculating vortices changes from four to five.

\section{Conclusion}

Two-dimensional incompressible flows over an open cavity are numerically investigated. The mode switching between non-oscillations, mode II, mode III and wake mode are simulated. The minimum length for initiation of self-sustained oscillations is $L / D=1.7$. The mode switching from mode II to mode III occurs between $L / D=3.1$ and $L / D=3.2$. The Strouhal number variation is consistent with the experimental results of Knisely and Rockwell [3]. The mode switching from the mode III to the wake mode occurs between $L / D=3.6$ and $L / D=3.7$. The time-averaged flow fields for ratios indicate that transitions from non-oscillations to mode II and from mode II to mode III occur when number of recirculating vortices change.

\section{Acknowledgements}

This research was partly supported by JSPS KAKENHI Grant Number 18560160.

\section{References}

[1] Rockwell, D. and Naudascher, E. (1978) Review-Self-Sustaining Oscillations of Flow past Cavities. ASME Journal of Fluids Engineering, 100, 152-165. https://doi.org/10.1115/1.3448624

[2] Sarohia, V. (1977) Experimental Investigation of Oscillations in Flows over Shallow Cavities. AIAA Journal, 15, 984-991. https://doi.org/10.2514/3.60739

[3] Knisely, C. and Rockwell, D. (1982) Self-Sustained Low-Frequency Components in an Impinging Shear Layer. Journal of Fluid Mechanics, 116, 157-186. https://doi.org/10.1017/S002211208200041X

[4] Gharib, M. (1987) Response of the Cavity Shear Layer Oscillations to External Forcing. AIA A Journal, 25, 43-47. https://doi.org/10.2514/3.9576

[5] Gharib, M. and Roshko, A. (1987) The Effect of Flow Oscillations on Cavity Drag. Journal of Fluid Mechanics, 177, 501-530. https://doi.org/10.1017/S002211208700106X

[6] Rowley, C.W., Colonius, T. and Basu, A.J. (2002) On Self-Sustained Oscillations in TwoDimensional Compressible Flow over Rectangular Cavities. Journal of Fluid Mechanics, 455, 315-346. https://doi.org/10.1017/S0022112001007534

[7] Rubio, G., De Roeck, W., Baelmans, M. and Desmet, W. (2007) Numerical Identification of 
Flow-Induced Oscillation Modes in Rectangular Cavities Using Large Eddy Simulation. International Journal for Numerical Methods in Fluids, 53, 851-866.

https://doi.org/10.1002/fld.1310

[8] Armfield, S. and Street, R. (2002) An Analysis and Comparison of the Time Accuracy of Fractional-Step Methods for Navier-Stokes Equations on Staggered Grids, International Journal for Numerical Methods in Fluids, 38, 255-282. https://doi.org/10.1002/fld.217

[9] Kim, J. and Moin, P. (1985) Application of a Fractional Step Method to Incompressible Navier-Stokes Equations. Journal of Computational Physics, 59, 308-323. https://doi.org/10.1016/0021-9991(85)90148-2

[10] Morinishi, Y., Vasilyev, O.V. and Ogi, T. (2004) Fully Conservative Finite Difference Scheme in Cylindrical Coordinates for Incompressible Flow Simulations. Journal of Computational Physics, 197, 686-710. https://doi.org/10.1016/j.jcp.2003.12.015

Submit or recommend next manuscript to SCIRP and we will provide best service for you:

Accepting pre-submission inquiries through Email, Facebook, LinkedIn, Twitter, etc. A wide selection of journals (inclusive of 9 subjects, more than 200 journals)

Providing 24-hour high-quality service

User-friendly online submission system

Fair and swift peer-review system

Efficient typesetting and proofreading procedure

Display of the result of downloads and visits, as well as the number of cited articles

Maximum dissemination of your research work

Submit your manuscript at: http://papersubmission.scirp.org/

Or contactojfd@scirp.org 